\title{
La reinvención neoliberal de la inequidad en Chile. El caso de la salud
}

\author{
Neoliberal reinvention of inequality in Chile: \\ the case of the health sector
}

Maria Eliana Labra 1

\footnotetext{
1 Departamento de Administração e Planejamento em Saúde, Escola Nacional de Saúde Pública, Fundação Oswaldo Cruz. Av. Leopoldo Bulhões 1480, Rio de Janeiro, RJ 21041-210, Brasil. labra@ensp.fiocruz.br
}

Abstract This paper examines the main changes in the Chilean health system from the 1920s to the present, identifying contradictions, limits, and successes in terms of policy outcomes. Taking equity as the central theme, the analysis focu ses on decisions made from the mid-1920s until 1973 in moving toward socialized medicine, with the sudden interruption of this process by the military coup in 1973. The author then discusses the set of regressive measures taken by authoritarian neoliberalism, with the help of Pinochet, to insert health into the market economy. FinalIy, the article analyzes efforts made by democratic admi nistrations since 1990 to redress the legacy of socioeconomic inequalities, focusing on the commitment to rebuild the health care system with a new basis in equity, solidarity, and people's participation.

Key words Equity; Health System; Health Policy; Social Security Financing

Resumen El artículo expone los principales cambios en el sistema de salud de Chile desde los años 20 hasta la actuali dad, señalando las contradi cciones, las limitaci ones y los avances en cada coyuntura política. Tomando como eje el tema de la equidad, la exposición examina las decisi ones tomadas desde mediados de 1920 hasta 1973 para avanzar en di rección a la socialización de la medicina y la abrupta interrupción de ese proceso con el golpe militar de 1973. En seguida, se aborda el conjunto de medi das regresivas tomadas por el neoliberalismo autoritario para insertar la salud en la economía de mercado. Por último, se analizan los esfuerzos emprendidos por los gobiernos democráti cos desde queasumieron el poder, en 1990, en el sentido de corregir las desi gual dades socio-económi cas heredadas, enfocando el compromiso de reconstruir el sistema médico asi stencial bajo nuevas bases asentadas en los principios de equi dad, solidaridad y participación.

Palabras clave Equidad; Sistema de Salud; Política de Salud; Financiamiento de la Seguridad Social 


\section{Introducción}

En Chile, la preocupación con la atenuación de las desigualdades en los niveles de salud de la población y en el acceso a los cuidados médicos y sanitarios acompañó el desarrollo de los servicios asistenciales desde los años 20 hasta el golpe militar de setiembre de 1973. Durante el sistema democrático vigente hasta entonces, esa trayectoria fue pautada por políticas de saIud impregnadas por un fuerte sesgo de clase. No obstante, avances notables fueron alcanzados en la expansión del derecho a la salud bajo las banderas doctrinarias de la medicina social, dejando un legado histórico que, en los años 80 , el neoliberalismo autoritario no consiguió extinguir en el grado pretendido. De forma tímida y oscilante, los gobiernos democráticos, desde que asumieron el poder en 1990, han adoptado medidas para superar las desigualdades sociales heredadas, destacando la declarada voluntad política del actual gobierno de realizar una reforma en la salud basada en principios como equidad, universalidad y participación. El objetivo de esta exposición es examinar esos cambios, teniendo como telón de fondo el contexto político de las transformaciones en el sistema de salud chileno y los grados de equidad resultantes en cada coyuntura de decisión, destacando los esfuerzos emprendidos por la coalición gobernante que inaugura, en 1990, la "neodemocracia” (Schmitter, 1993) en el sentido de atenuar las disparidades sociales heredadas de la dictadura, en particular en el sistema médico sanitario.

Las políticas de salud en

el Estado de compromiso

La creación del Seguro Obrero Obligatorio chileno, en 1924, introdujo por primera vez en el país un régimen previsional de repartición financiado con contribuciones tripartitas, destinado a proteger a los trabajadores "manuales" del mercado formal contra los riesgos de invalidez, vejez, enfermedad y accidentes de trabajo. Dejó de lado, por lo tanto, el estrato más pudiente por considerarse que contaba con los medios para asumir los costos de esos riesgos, así como los empleados en los sectores público y privado, para los cuales legislación paralela inauguró un esquema de previsión de capitalización individual. La atención médica para los beneficiarios del Seguro Social era prestada en ambulatorios propios y, mediante convenios, en los hospitales beneficientes que, desde la Colonia, atendían a los indigentes. Mientras que ese complejo fue el objeto de las transformaciones que serán aquí examinadas, el régimen de protección propio de las Fuerzas Armadas y Policías fue mantenido sin grandes alteraciones hasta la actualidad.

Tal como en otros países de América Latina, la instauración del sistema de protección social en Chile coincide con el paso de la dominación oligárquica para el Estado Moderno, que transcurre en los tumultuosos años 20, cuando ya se definía en el escenario político un espectro político partidario claramente dividido entre fuerzas conservadoras, de izquierda y de centro, que va a constituirse en el eje de las relaciones estado-sociedad. En esa difícil coyuntura, el Congreso Nacional aprobó, bajo presión de los militares y sin mayor discusión, la legislación social que la coalición liberal había postulado en la campaña electoral. Más aún, en 1925 fue promulgada una Constitución liberal moderna que, además de asegurar los derechos civiles, políticos y sociales, estableció como deber del Estado mantener un servicio nacional de salud pública, bajo la orientación de John Long, asesor de la Fundación Rockefeller. A este propósito, cabe recordar que en aquella época los Estados Unidos, a través de esa institución y de la Oficina Sanitaria Panamericana (actual OPS - Organización Panamericana de la Salud), estaban empeñados en inducir a los gobiernos latinoamericanos a adoptar un código sanitario uniforme y a organizar la salud pública de forma centralizada y dirigida por un estamento médico especializado - los sanitaristas o salubristas - inicialmente capacitado en Johns Hopkins y más tarde en cursos o escuelas de higiene y salud pública de los propios países (Labra, 1985).

La discriminación establecida con el Seguro Social chileno entre trabajadores y empleados derivó en una pugna histórica de los sectores progresistas liderados desde comienzos de Ios años 30 por el médico socialista Salvador Allende, parlamentario y ministro del área social (1939-1941) y, más tarde, Presidente de la República. Allende se debatió por la integración de todos los servicios de salud en una única institución, propugnando además políticas destinadas a corregir las disparidades entre regiones y clases sociales, a partir del principio de que la salud es un derecho de todos y un deber del Estado y que la situación de salud es determinada, ante todo, por factores como salarios dignos, vivienda, alimentación, educación, diversión y cultura (Allende, 1939). Los embates en torno a estas cuestiones vinieron a luz con gran fuerza durante la efímera “República Socialista" de mediados de 1932, cuando las iz- 
quierdas hicieron de la salud un caballo de batalla en la lucha por transformaciones económicas y sociales. Influenciados por el sistema soviético, grupos médicos socialistas pregonaron una verdadera "dictadura sanitaria" que diseminaría "usinas sanitarias" por todo el país, formando un conjunto universal, integrado, eficiente y humano, dirigido por un "estado mayor técnico" que tendría en el planeamiento centralizado su herramienta principal para llevar adelante el revolucionario proyecto.

Esos eventos recrearon, en la esfera de la salud, la histórica lucha político partidaria entre tres corrientes ideológicas, doctrinarias y programáticas que dividió a los profesionales y cuyos vestigios todavía persisten: la derecha conservadora, defensora de la medicina preventiva universal y de la asistencia médica estatal sólo para los pobres y desvalidos; el centro, favorable a la manutención de la asistencia médica previsional conforme el modelo de la Organización Internacional del Trabajo (OIT) de protección a los asalariados, separado de la salud pública; y la izquierda, partidaria de un sistema de salud único, integrado y universal. Entre tanto, por la propia naturaleza del Estado de compromiso, las reformas del sistema de protección social resultaron en un híbrido de essas corrientes, ya que los proyectos de ley habrían de pasar por la arena parlamentaria.

Debido a esos bloqueos, las reivindicaciones en pro de la transformación del esquema de protección social en un sistema más amplio y equitativo fueron sólo parcialmente conquistadas cuando, en 1952, mediante artificios propiciados por las reglas del juego parlamentario, fue insertado en la ley que modificaría el Seguro Social un artículo creando el Servicio Nacional de Salud (SNS). Tal innovación tuvo su fundamento doctrinario en el hecho de que la capitalista Inglaterra, en 1948, había cimentado la Seguridad Social conforme el binomio seguridad económica y biológica asegurado por dos instituciones diferentes, quedando la segunda por cuenta del National Health Service (NHS) que, con recursos del Tesoro, daría cobertura universal a todos los ciudadanos. En Chile, sin embargo, la tentativa de imitar el modelo inglés se vio perjudicada en la medida que el SNS quedó injertado en el sistema previsional. De hecho, después de 11 años de discusión en el Congreso Nacional, no fue posible negociar la universalización sino tan sólo la ampliación y mejoría de los beneficios del Seguro Social, permaneciendo el financiamiento tripartito y los derechos sociales restringidos a los obreros urbanos, lo que demostró la fuerza política que todavía detenía la derecha latifundista. Asimis- mo, el SNS, aunque unificó todos los servicios asistenciales y sanitarios del país, mantuvo la misma cobertura para obreros e indigentes (Chile, 1952). Este resultado se debió a las condiciones impuestas por la coyuntura política: después de las elecciones parlamentarias de 1949 y hasta 1952, cuando concluye el último gobierno del Frente Popular, desde 1938 en el poder, la correlación de fuerzas en el Parlamento pasó a ser dominada por conservadores y liberales que bloquearon una reforma más generosa en la seguridad social. Por las mismas circunstancias, no fue posible aprobar el SNS como un proyecto separado, de modo que quedó sujeto a las mismas limitaciones del Seguro Social, además de preservar las diferencias de clase.

Sin embargo, no solamente el SNS constituyó el segundo intento de su género en el mundo capitalista después del NHS, sino que emergió como un modelo paradigmático en América Latina debido a características como ingeniería institucional, competencia técnica, organización capilar territorial mediante "zonas de salud" y la adopción inédita de métodos de planeamiento y programación que hicieron escuela en el continente. La nueva institución integró todos los programas de salud pública y de medicina preventiva, hasta entonces dispersos en diversas reparticiones, así como los servicios médicos del Seguro Social, englobando también los hospitales beneficientes del país, que se constituyeron en centros de excelencia de la medicina chilena. Los cuadros técnicos del SNS, oriundos de la Escuela Nacional de Salubridad (1943), formaron una élite altamente capacitada, a la par que los dirigentes de la institución pasaron a concentrar gran poder, al punto de ofuscar al propio ministro de Salud.

Cabe aún señalar que, por una serie de factores históricos y políticos, en Chile los profesionales de la medicina se convirtieron en actores de importancia central del sector e incluso a nivel nacional, en particular a partir de la fundación del Colegio Médico en 1948, y que Allende pasó a presidir. En efecto, a medida que avanzaba la discusión de la reforma de la previsión social y la fusión de los servicios de salud, la profesión médica luchó por una asociación de representación monopólica y demás prerrogativas de status público, semejante a la de los abogados. Gracias a esa poderosa organización, pudieron negociar la promulgación de un estatuto especial que los convirtió en funcionarios públicos con una serie de regalías en relación al resto de la Administración Pública (Chile, 1951). Asimismo, los asientos del Consejo Nacional de Salud incluido en el diseño del SNS pasaron a ser ocupados casi todos por 
médicos, a pesar de haber sido pensado como una arena de participación democrática en la cual todas las partes estarían igualitariamente representadas.

Por último, debe ser subrayado que, por razones igualmente históricas, en Chile el segmento médico asistencial privado se desarrolló de forma marginal. Con efecto, hasta comienzos de los años 80, sobrevivió gracias a convenios con el Servicio Médico Nacional de Empleados (SERMENA), que propiciaba a su contingente de beneficiarios - cerca de un $20 \%$ de la población en los años 60 - principalmente consultas médicas, exámenes complementarios y atención al parto, con base en la libre elección entre los profesionales y establecimientos inscritos en el SERMENA.

Como veremos adelante, es contra esos centros de poder casi autónomos y la ausencia de relaciones de mercado en la salud que los neoliberales van a arremeter con toda su fuerza.

En 1960, la población ascendía a 7,4 millones, la mayoría en precaria situación económica puesto que el $72 \%$ de la Población Económicamente Activa ganaba menos de un sueldo vital. Por otra parte, a pesar del sesgo de clase en el acceso a los servicios médicos del SNS, este cubría al $70 \%$ de la población porque las personas sin derecho legal utilizaban los servicios por medio de expedientes informales, en especial los empleados de renta media y baja y sus familiares. La infraestructura hospitalaria era casi toda estatal ya que el SNS contaba con 36.385 camas mientras que el sector privado tenía solamente 1.403 camas, la mayoría obstétricas (Ministerio de Salud Pública, 1970).

La no resolución del problema del acceso universal a la atención en el SNS, además del déficit financiero crónico causado por el exceso de demanda, provocó innumerables antagonismos que se arrastraron hasta 1968, cuando, bajo el gobierno demócrata cristiano, un nuevo esfuerzo es emprendido para crear un sistema de salud universal. No obstante, el respectivo proyecto de ley fue nuevamente mutilado. Entre otras razones, merece destacar la presión de los médicos por la expansión del mercado privado de trabajo como medio para incrementar las rentas, acompañando así el movimiento continental en favor de un status más elevado para la profesión. En esta lucha, buena parte de los médicos chilenos se alió a las asociaciones de empleados en la reivindicación intransigente de este segmento de no ser equiparado a los obreros e indigentes en la atención en el SNS. Como en esa época el prestigio del gobierno demócrata cristiano, que había asumido en 1964 bajo el lema "revolución en liber- tad", ya estaba erosionado, no fue posible alcanzar avances mayores. De ello resultó una ley muy peculiar que insertó el esquema (y recursos) del SERMENA en el SNS, estableciéndose, así, condiciones especiales para que los empleados y funcionarios públicos fuesen atendidos mediante libre elección del profesional, copagos por acto médico y atención en horario distinto del destinado a la población beneficiaria tradicional (Chile, 1968). Esto es, se instituyó dentro del SNS una atención diferenciada, con dos formas de gestión que desorganizaron la unicidad existente y reforzaron la discriminación de clase, además de no resolver de manera efectiva la cuestión financiera.

A su vez, Salvador Allende, al asumir la Presidencia de la República, a finales de 1970, empuñó con determinación la bandera del Sistema Unificado de Salud, a ser financiado solamente con recursos del Tesoro Nacional, que acabaría con las discriminaciones existentes, incluiría la participación comunitaria y, mediante un conjunto de políticas sociales redistributivas y la mejoría de la calidad de los servicios, incentivaría la elevación de las condiciones de vida, la equidad en el acceso y la calidad en la asistencia. A este proyecto se opuso tenazmente la mayoría de los médicos. Más aún, en el clima de polarización ideológica reinante, el Colegio Médico terminó apoyando a los sectores reaccionarios en la petición de renuncia del colega presidente.

El cruento golpe militar de septiembre de 1973 puso fin a la senda democrática que a lo largo de 140 años se venía ensanchando en el país, abortando toda y cualquier iniciativa progresista, hundiendo a la nación en el terror, en la inseguridad y en la retracción de tantas conquistas alcanzadas en términos de derechos políticos, civiles y sociales.

\section{Las reformas del neoliberalismo} autoritario

En otro lugar analizamos con detalle el pionero experimento neoliberal emprendido en Chile desde mediados de los años 70 en adelante (Labra, 1995; 1997; 2000). Para fines de esta exposición, cabe apenas registrar que, bajo el dogma de la primacía del mercado y de la falencia del Estado Benefactor, el núcleo hegemónico comandado por Pinochet y economistas neoliberales implementó en muy corto tiempo (19781981) un audaz proyecto privatizador que atribuyó al Estado un papel apenas subsidiario de la iniciativa privada. Tal proyecto contó con el apoyo del grupo ultraderechista de médicos que 
de un tomó por asalto el Colegio Médico cuando se produjo el golpe de Estado, avocándose de inmediato a elaborar un plano que ya contenía las líneas maestras de las futuras reformas estructurales en la previsión social y la salud (MINSAL, 1975).

Tocando solamente el campo que aquí interesa, los fondos del seguro social acumulados por los afiliados con base en los aportes tripartitos fueron perentoriamente transferidos a Administradoras de Fondos de Pensiones (AFP), que son sociedades mercantiles privadas, lucrativas y competitivas, formadas con capitales nacionales y de holdings internacionales. A pesar de las graves restricciones de la época impuestas por las crisis internacionales, la viabilidad económica del nuevo sistema fue posibilitada por la maciza transferencia de recursos desde los demás sectores sociales hacia la previsión social, como muestra la Tabla 1.

Paralelamente, las jubilaciones y pensiones de los que se inscriben en Ias AFP pasan a ser financiadas solamente con la contribución obligatoria del $10 \%$ del salario del afiliado (excepto pensiones mínimas concedidas por el Estado), siendo optativos los aportes adicionales para los diversos seguros ofrecidos en la cartera de servicios de aquellas.

Como sintetiza Elter (1999), el sistema de ahorro individual obligatorio de las AFP no es un instrumento idóneo para promover una protección social en los riesgos asociados a la vejez, a la invalidez y a la muerte; no presenta condiciones de sustentación a largo plazo; carece de cualquier efecto redistributivo; $y$, afecta específicamente a la mujer trabajadora debido a la discriminación directa legalmente establecida. En la misma línea, Zapatta (1999) afirma que los inmensos recursos acumulados por las AFP, en torno a 33 mil millones de dólares, constituyen una herramienta financiera de primera categoría, señalando que el sistema en sí, además de ser engañoso y lleno de ambigüedades, ha permitido "el más gigantesco enriquecimiento, para unos pocos, del que se tenga recuerdo en nuestra historia". No obstante las constatadas desigualdades de este modelo, experimentado de forma pionera en Chile, varios países latinoamericanos lo han adoptado, no por la vía de la fuerza, sino mediante decisiones democráticamente tomadas en los parlamentos, a pesar de fuerte resistencia popular, como testifican los casos de Colombia, de Argentina, de Uruguay o de Bolivia.

Con la privatización del sistema previsional, el sector salud quedó libre para que se introdujesen reformas estructurales totalmente opuestas a los rumbos anteriores, instituciona-
Tabla 1

Gasto fiscal social, 1974-1989.

\begin{tabular}{ccccc}
\hline Año & $\begin{array}{c}\text { Previsión social } \\
\%\end{array}$ & $\begin{array}{c}\text { Salud } \\
\%\end{array}$ & $\begin{array}{c}\text { Educación } \\
\%\end{array}$ & $\begin{array}{c}\text { Vivienda } \\
\%\end{array}$ \\
\hline 1974 & 21,0 & 17,5 & 38,7 & 17,5 \\
1980 & 29,5 & 13,9 & 33,5 & 5,5 \\
1984 & 39,2 & 7,8 & 24,9 & 3,9 \\
1989 & 49,1 & 6,9 & 22,0 & 7,7 \\
\hline
\end{tabular}

Fuente: Rozas (1997).

lizándose un nuevo principio axiológico: la inequidad como un fenómeno "natural" que solamente la libre competencia mercantil y el esfuerzo individual podrían corregir.

En breve síntesis, las transformaciones fueron las siguientes: fortalecimiento del Ministerio de Salud (MINSAL), que asume el papel rector político-técnico del sector; extinción del SNS, creándose en su lugar el Sistema Nacional de Servicios de Salud (SNSS), regionalizado en 27 servicios; y, la municipalización de la Atención Primaria, que acarreó infinidad de problemas ( pérdida de status de funcionarios públicos del personal; falencia de los centros de salud; desorganización de los registros de información epidemiológica; grave congestionamiento de la atención, etc.), exacerbados por el desmantelamiento de los hospitales públicos, el despido de cuadros y la depreciación de los salarios. Fue aún establecida la separación entre las funciones normativas (MINSAL), las ejecutivas (servicios regionales) y las financieras, estas a cargo del FONASA (Fondo Nacional de Salud), nueva y poderosa autarquía del MINSAL estrechamente ligada a las áreas económicas del gobierno, encargada de la administración de los recursos, del control de los flujos financieros y de la gestión del régimen de libre elección.

Otras dos iniciativas, más decisivas aún, fueron la introducción de un segmento comercial de planos privados de salud, inexistentes en Chile hasta entonces, y el radical cambio en la forma de financiamiento.

La primera iniciativa fue la creación de las Instituciones de Salud Previsional o Isapre, mediante decreto-ley de 1981. Las Isapre son empresas privadas lucrativas cuya función es realizar la intermediación financiera en la compra y venta de planos de salud, aunque, excepcionalmente, algunas poseen establecimientos asistenciales. Gracias a incentivos fiscales y otros artificios mencionados adelante, el des- 
pegue de esta industria fue rápido: el número de Isapre pasó de 6 en 1981 a 23 en 1989, abarcando al $21 \%$ de la población, el $10 \%$ de la cual está cubierta por contratos colectivos. No obstante, la pretensión de que las I sapre substituyeran a los servicios públicos se realizó en grado mucho menor del esperado puesto que, después de un efímero auge a mediados de los años 90 , actualmente su cobertura está estancada en niveles próximos al $20 \%$ de la población.

La segunda medida tiene que ver con los cambios introducidos en la financiación, que merecen atención especial puesto que viabilizaron la determinación del neoliberalismo autoritario de implementar una medicina para ricos y otra para pobres. Como se ha dicho, el ex-SNS se financiaba con parte de las contribuciones tripartitas del Seguro Social. Mas, debido a la insuficiencia de estos recursos, el Tesoro terminó asumiendo casi dos tercios del presupuesto, de modo que la carga se distribuía de forma solidaria entre la población. Con la reforma, los patrones quedaron exentos de contribución mientras que las personas, después de sucesivos aumentos, desde 1986 en adelante pasaron a contribuir con un $7 \%$ de la renta tributable. Tal como en el caso de las AFP, la equiparación de los aportes se fundamentó en el argumento de que como en el mercado todos los individuos tienen igual oportunidad, no debe existir discriminación en el libre acceso a la oferta de servicios de salud, sean públicos o privados. Como manifestó el entonces ministro José Piñera, mentor de la reforma de la previsión social, la contribución igualitaria acabaría con la lucha de clases (Piñera, 1991). Sin embargo, como veremos en seguida, los mecanismos introducidos para restringir el acceso gratuito a los cuidados médicos solamente restablecieron de forma más sofisticada las discriminaciones entre los ciudadanos.

En cuanto a los recursos fiscales, la idea original era que el aporte del Estado fuese menguando hasta hacerse marginal cuando las familias asumiesen gran parte del costo; pero esta meta quedó bien lejos de lo pretendido, como muestra la Tabla 2 más adelante. Con el aumento de la contribución y una serie de incentivos fiscales concedidos a las Isapre, se buscó sobretodo impulsar su desarrollo. Entre los subsidios vale mencionar el aumento de la contribución para la salud de los jubilados y pensionistas del 1,5\% al 7\%, la exención de pagar el auxilio maternidad, que se mantuvo a cargo del Estado, y la concesión de un 2\% adicional para que grupos de trabajadores de baja renta pudiesen comprar un plan de salud.
Resucitando la vieja idea conservadora de que solamente los pobres e indigentes debían recibir protección social del Estado, el acceso a la asistencia médica quedó así estratificado: (a) los segmentos con mayores rendimientos deben comprar planes de salud en el mercado según la opción individual; (b) los sectores medios pueden optar por el régimen de libre elección del FONASA, con copagos variables según el nivel del profesional; (c) los estratos de bajas rentas son atendidos en los servicios estatales de forma gratuita mediante comprobante de la condicióde pobreza, pagando, en caso contrario, un porcentaje de acuerdo a los ingresos.

Es importante mencionar a este respecto que las condiciones establecidas en la forma de acceso a la atención médica destruyeron toda y cualquier solidaridad en cuanto al financiamiento global, fundamentalmente porque las personas que optan por afiliarse a una Isapre quedan exentas de contribuir para el FONASA. Y como los grupos de más altas rentas son los que migran al segmento privado, se produce el Ilamado efecto "descreme" en el financiamiento del sistema público. En resumen, las personas que acuden al SNSS terminan financiando a los estratos más ricos (Figura 1).

La reforma en general y la estratificación del acceso consolidaron, en realidad, dos segmentos desconexos, el público y el privado. En consecuencia, las formas de acceso reflejan la histórica división entre tres estratos distintos a la hora de buscar cuidados médicos: los más ricos (Isapre), los sectores medios (libre elección) y la mayoría de menores recursos (modalidad institucional). Estas discriminaciones tuvieron sus fundamentos en el cambio del patrón de financiamiento. De hecho, como puede ser observado en la Tabla 2, en el período los aportes fiscales disminuyeron por la mitad mientras el conjunto de aportes de la población más que se duplicó.

Los efectos sociales de la política económica neoliberal no fueron menos nefastos: la proporción de hogares en condiciones de pobreza que, en 1970 se estimaba en un 17\%, en 1987 alcanzaba a un 44,6\% (Fazio, 1996). Paralelamente, los indicadores de morbilidad se alteraron negativamente, sobre todo trastornos mentales causados por los tantos traumas y el desempleo sufridos por los chilenos, enfermedades de la infancia derivadas de la miseria y muchos otros daños que habrían sido evitados si los servicios asistenciales hubiesen funcionado adecuada y oportunamente (Medina, 1989). No obstante, como será examinado adelante ( Tabla 3), se observó la paradoja de una continuada mejoría de los indicadores básicos de salud. 
Debe aún ser señalado que, con el argumento de que el corporativismo conspira contra la libre competencia en el mercado, los neoliberales asumieron la misión de desmontar el andamio asociativo que desde mediados del siglo 19 se había construido en el país. En efecto, un rasgo típico da cultura política chilena fue la tendencia a la formación de organizaciones de representación monopolística en las esferas del mundo empresarial, sindical y profesional, imbricadas con los partidos políticos. Esto implicaba afiliación y contribución obligatorias y, en el caso de los profesionales liberales, prerrogativas como el control ético de los pares. Tal desmonte fue practicado decretándose la libre asociación y la substitución de las organizaciones vigentes por "asociaciones gremiales" de afiliación voluntaria. En seguida, se permitió a las colectividades escoger nuevos dirigentes y reiniciar las actividades, hasta entonces prohibidas. No cabe aquí profundizar en este importante tópico y sus consecuencias en términos de la dispersión de las fuerzas del mundo laboral, sino sólo tocar en sus reflejos en el Colegio Médico, que por este acto vio sepultadas las conquistas alcanzadas por la profesión en 1948. Con mucha dificultad, la Orden consiguió renacer eligiendo en 1981 nuevos dirigentes comprometidos con la lucha contra la dictadura; pero la cohesión de la profesión nunca fue la misma debido a la segmentación del mercado de trabajo y consiguiente fragmentación de los intereses corporativos. Dígase de paso que, por su parte, las Isapre se organizaron en una poderosa asociación, constituyéndose así en el interlocutor privilegiado de las autoridades y avasallando al Colegio Médico que, desde su fundación, había dominando los escenarios de opinión y las mesas de decisión. Las intermediarias fueron aún más fortalecidas con la creación de la Superintendencia de Isapre, subordinada al MINSAL, para regularlas pero, en realidad, fue decretada cuando la dictadura dejó el gobierno (marzo de 1990) como forma de mantener las reglas del juego. Inversamente, se evidencia un débil poder negociador de los afiliados a las I sapre frente a las selectividades y discriminaciones estipuladas en los numerosos tipos de planes, que afectan especialmente a los ancianos y a las mujeres en edad fértil.

En el plano político, la dictadura militar había promulgado en 1980 la nueva Constitución que debería entrar en pleno vigor en octubre de 1988 cuando el pueblo, mediante plebiscito, decidiría la continuidad o no de Pinochet en el gobierno. Como sabemos, después de difíciles negociaciones, la democracia cristiana y los so-
Tabla 2

Fuentes de financiamiento de la salud 1974-1989.

\begin{tabular}{lcccc}
\hline Fuente de recursos & $\begin{array}{c}1974 \\
\%\end{array}$ & $\begin{array}{c}1980 \\
\%\end{array}$ & $\begin{array}{c}1989 \\
\%\end{array}$ & $\begin{array}{c}\text { Variación } \\
1989-1974 \\
\%\end{array}$ \\
\hline Fisco & & & & -49 \\
Contribuciones & 16 & 55 & 35 & 180 \\
Copagos & 10 & 13 & 45 & 50 \\
Otros & 6 & - & 15 & -17 \\
Total & 100 & 100 & 100 & - \\
\hline
\end{tabular}

Fuente: MINSAL (1992).

Figura 1

Esquema de "opciones" de acceso a la atención de la salud.

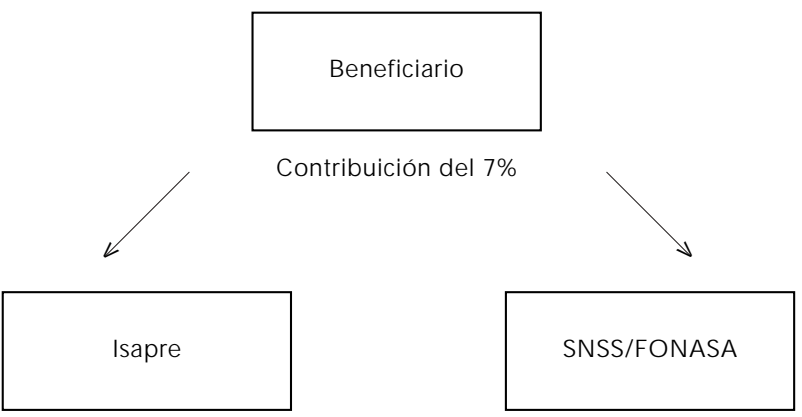

$7 \%+$ adicional opcional

$7 \%+$ copagos obligatorios
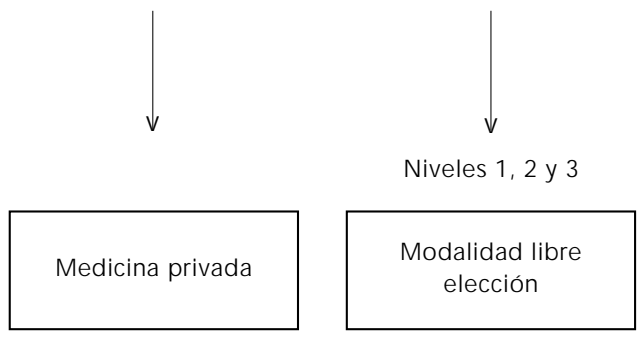

Grupos A, B, C y D

Elaboración propia.

Isapre = Institución de Salud Previsional; SNSS = Sistema Nacional de Servicios de Salud; FONASA = Fondo Nacional de Salud.

cialistas consiguieron formar la coalición Concertación de Partidos por la Democracia y derrotar al dictador en ese memorable acto cívico. Durante el año 1989 la Concertación entabló acuerdos con el gobierno autoritario para cambiar las partes más represivas de la Carta, pero no logró alteraciones en cuestiones clave como las relativas al sistema político y electoral, al 
Tabla 3

Chile: evolución del nivel de salud, 1974, 1990, 1998.

\begin{tabular}{lrrr}
\hline Indicadores & 1974 & 1990 & 1998 \\
\hline Población total (millones) & 10,0 & 12,9 & 15,2 \\
Mortalidad generala & 7,7 & 6,0 & 5,4 \\
Mortalidad infantilb & 65,2 & 16,0 & 10,3 \\
Mortalidad neonatalb & 26,1 & 8,5 & 5,9 \\
Mortalidad maternab & 1,2 & 0,4 & 0,2 \\
Natalidada & 24,2 & 23,4 & 18,3 \\
Atención profesional al parto & 86,0 & 98,8 & 100,0 \\
\% Niños desnutridos (0-5 años) & 15,5 & 8,6 & 5,1 \\
Esperanza de vida al nacer & 58,5 & 74,4 & 75,2 \\
\hline
\end{tabular}

a por mil habitantes.

b por mil nacidos vivos.

c 28 días-1 año, por mil nacidos vivos

Fuentes: Lavados (1984), M IN SAL (1991, 2001a) y Raczynski (1991)

funcionamiento de los poderes públicos y a las atribuciones de las Fuerzas Armadas en especial. A esto se debe que la "neodemocracia" chilena constituya un régimen que permanece tutelado por los militares y cuyo cambio depende de una reforma constitucional imposible dado el elevado quórum parlamentario requerido, dificultado más aún por la cuota de senadores "designados" que la misma Carta estipuló, entre otros dispositivos que la maniatan. En todo caso, las elecciones de finales de 1989 dieron la victoria a las fuerzas de la Concertación que, desde marzo de 1990, se mantienen en el gobierno, aunque haya cambiado el matiz político del presidente: en las elecciones de 1989 y las de 1993 ganó la primera magistratura un demócrata cristiano mientras que en el pleito de 1999 la victoria fue para un socialista, el actual Presidente Ricardo Lagos.

En síntesis, la dictadura cumplió en buena medida su misión de "refundar" la nación mediante transformaciones estructurales dictadas bajo la inspiración de los “Chicago boys”, dejando un legado de iniquidades en las áreas sociales, mientras que en el plano económico, a los pocos, la nueva sociedad de mercado se acomodaba a los imperativos de la globalización y a la tiranía de la especulación financiera, consiguiéndose un precario equilibrio interno que los gobiernos democráticos debieron mantener, bajo pena (en teoría) de desorganizar la reconversión productiva que había insertado al país en las redes internacionales de comercio. Todo eso fundamentado en la nueva cultura del individualismo, del consumismo y de la desconfianza en la política y en los políticos.
Esperanzas e incertidumbres en la "neodemocracia"

La Concertación de Partidos por la Democracia se ha mantenido al frente en la difícil tarea de conciliar el modelo económico neoliberal con la atenuación de las desigualdades heredadas, en un contexto de serias restricciones institucionales derivadas de las "amarras" constitucionales que aún hoy impiden reformas legales defondo.

El sector salud, en el retorno a la democracia, mostró altos grados de ingobernabilidad debido a la acumulación de demandas de los funcionarios de los servicios y de la sociedad. A lo largo de la década de los 90, no obstante, se observaron progresos en la adopción de medidas para corregir las distorsiones más flagrantes, aunque manteniéndose el mismo formato del sistema de salud - fracturado, estratificado, inicuo y deficitario. En esta última parte haremos breves consideraciones ilustradas con datos comparativos sobre los avances alcanzados y las dificultades enfrentadas por la Reforma de la Salud actualmente en debate.

En el plano de los nuevos valores éticos que fueron delineados para el sector por la Concertación, y también por el Colegio Médico, resaltan: la salud como derecho universal, acceso igualitario, financiamiento solidario, efectividad y eficiencia en la gestión, participación y, lo más importante, la equidad, que resume los principios anteriores y enfatiza la prestación de cuidados buscando el equilibrio en las condiciones de grupos distintos en términos socio-económicos, de riesgo, de género y geográficos. Tales intenciones tuvieron una primera traducción en el proyecto piloto del MINSAL, Enfoque de Equidad en Salud, de fines de 1991, focalizado en 45 municipios, de los 340 del país, totalizando 1,2 millones de personas, cuya mortalidad infantil era más alta (Montoya et al., 1997).

Como ese proyecto tuvo saldos positivos en términos de la mejoría de los indicadores de salud (en particular mortalidad infantil y enfermedades asociadas al alcoholismo), en 1994 el MINSAL definió la equidad como un principio básico de las políticas, tomando varias iniciativas tópicas en el ámbito de la ampliación de la cobertura del SNSS. Por ejemplo, primero, pasó la atención primaria a ser gratuita; y, recientemente, fue determinado prestar atención gratuita y otros beneficios a los mayores de 60 años inscritos en el FONASA, después de constatarse que, de un total de 1,3 millones de adultos mayores, solamente el $10,9 \%$ estaba afiliado a una Isapre. De la misma manera se 
procedió con el parto dado que sólo el 47,\% de las mujeres en edad fértil estaban inscritas en una Isapre (MINSAL, 1999).

Se subraya que medidas como esas, aliadas a factores como el alto precio y la baja cobertura de los planes de salud, la supresión legal del mencionado subsidio del $2 \%$ que se otorgaba a las Isapre, además del desempleo, Ilevaron a medio millón de personas a migrar a la modalidad institucional del FONASA por ser más barata que la de libre elección. Así, si a mediados de 1997 el porcentaje de la población beneficiaria de las I sapre ascendía al $27 \%$, hoy no pasa del 20\% (Novoa, 2001).

Por otro lado, objetivando mejorar la calidad de la atención, fue solucionado en buena parte el problema de las listas de espera en los niveles secundario y terciario. Se debe mencionar aún la adopción de la "Carta de Deberes y Derechos de los Pacientes" en muchos hospitales y cuya aplicación será obligatoria después de la promulgación de la respectiva ley en trámite en el Congreso Nacional. En el área de recursos humanos, fue editado un Estatuto de Atención Primaria, penosamente negociado, que hizo más atrayente trabajar en ese nivel. Así también, una vez más fue actualizado el antiguo estatuto para los profesionales de la salud pública (plan de cargos, carrera y salarios), concediéndose incluso aumentos salariales que paliaron las desmedradas remuneraciones.

Con relación a la estructura del financiamiento, la Tabla 4 muestra que hubo reversión de la tendencia anterior en la medida que el aporte fiscal para la salud tuvo un incremento substancial, aunque el nivel de 1974 ( Tabla 2) aún no haya sido alcanzado. En efecto, el cambio en el patrón de financiación en la década fue positivo: Ios recursos fiscales aumentaron cerca de un $50 \%$ mientras que las contribuciones, en particular los copagos, disminuyeron significativamente. Con respecto a la inversión, en el período aumentó seis veces en relación a la década de los 80 (MINSAL, 2001a). Por otra parte, perduran graves distorsiones. En 1973, el nivel histórico del SNS era de un 3,5\% del Producto Interno Bruto (PIB), pero la dictadura redujo esta cifra al 0,85\%. Actualmente, el país destina un $6,5 \%$ del PIB a la salud; mas, de ese total, un $4 \%$ es invertido en el sector privado, que atiende al $20 \%$ de la población, mientras que el sector público, responsable por más del $70 \%$ de la atención, se queda con sólo un $2,5 \%$ (González-Dagnino, 1999).

En cuanto a la cobertura del $70 \%$, tal porcentaje no refleja la realidad puesto que existen problemas que aumentan la demanda, y gravan el SNSS, principalmente debido al defi- ciente control del tipo de usuario de los servicios. Por ejemplo, se detectó que 500.000 personas que tenían ingresos fueron atendidas gratuitamente en calidad de indigentes. Asimismo, el $100 \%$ de las atenciones de urgencia y el $80 \%$ de las cirugías de baja, media y alta complejidad son realizadas en los servicios públicos; ello significa que hay beneficiarios de las Isapre pero estas no efectúan el respectivo resarcimiento al FONASA (Novoa, 2001).

Cabe aún apuntar que la mejoría de los indicadores de salud ha sido considerada espectacular. La Tabla 3 muestra la evolución de algunos de los indicadores de salud más importantes. Empero, esos indicadores esconden las desigualdades existentes en el país. Por ejemplo, la mortalidad infantil es de 42,2 por mil nacidos vivos en el municipio más pobre del país (en la región sur) mientras que es de 2,62 por mil en el municipio más rico (en Santiago) (MINSAL, 2001b).

De cualquier forma, los datos demuestran el continuo progreso en los indicadores básicos de salud debido a factores como la manutención de programas focalizados en el grupo materno-infantil; la erradicación del analfabetismo, la expansión de las redes de saneamiento básico, que cubre prácticamente al 100\% de la población urbana y, sobre todo, la permanencia del legado institucional y cultural dejado por el SNS en términos de compromiso con la res publica y con la prevención. Estos elementos ciertamente compensaron el aumento de la pobreza de los años 80.

A ese respecto, los gobiernos de la Concertación consiguieron disminuir el porcentaje de pobres, que era de casi el $45 \%$ en 1987 , al $22 \%$ en 1998, aunque en los hogares dirigidos por mujeres la magnitud de la pobreza alcanza al 56\% (Riquelme \& Barilari, 1997). Se debe considerar, así mismo, que la concentración de renta

Tabla 4

Fuentes de financiamiento de la salud, 1989-1998.

\begin{tabular}{lcccc}
\hline Fuente de recursos & $\begin{array}{c}1989 \\
\%\end{array}$ & $\begin{array}{c}1992 \\
\%\end{array}$ & $\begin{array}{c}1998 \\
\%\end{array}$ & $\begin{array}{c}\text { Variación } \\
1989-1998 \\
\%\end{array}$ \\
\hline Fisco & 35 & 46 & 52 & 49 \\
Contribuciones & 45 & 38 & 38 & -15 \\
Copagos & 15 & 5 & 9 & -40 \\
Otros & 5 & 11 & 1 & 80 \\
Total & 100 & 100 & 100 & - \\
\hline
\end{tabular}

Fuente: MINSAL (1993; 2001a). 
es extremadamente alta: mientras que el $20 \%$ de las familias de ingresos más altos obtiene el $60 \%$ de la renta nacional, el $20 \%$ de las familias de ingresos más bajos se queda con apenas el 4\% (González-Dagnino, 1999). Por lo tanto, las desigualdades en el plano económico social persisten; y, como tienen carácter estructural, continuarán afectando al bien estar de parte significativa de los chilenos.

Por último, se debe mencionar el compromiso asumido por el Presidente Lagos con la realización de una amplia y profunda Reforma de la Salud de cuyos principios generales destacan: "equidad en la atención; garantía de acceso sin cualquier tipo de exclusiones ni discriminaciones; $y$, solidariedad en el sistema de financiamiento, de modo que todos aporten según sus capacidades y se beneficien según sus necesidades". La propuesta oficial incluye reorganización institucional y se fundamenta en variadas premisas que tienen por objetivo promover la calidad, la efectividad y la eficacia dentro del SNSS. Al mismo tiempo, se argumenta que las Isapre presentan un déficit de protección y de transparencia; altos costos; regulación sanitaria insuficiente e ineficaz; e, incremento sostenido de los precios. La necesidad de superar las disparidades existentes es ilustrada con datos como los siguientes: el gasto anual per capita del sector público con salud es de US\$210 mientras que el sector privado gasta US\$ 500, a la par que, del total de horas médicas, $66,5 \%$ corresponden a la atención en el sector privado aunque este cubra a menos de un tercio de la población (MINSAL, 2001c).

La Reforma ha sido muy discutida por los diferentes sectores interesados. No obstante, está lejos de presentar propuestas concretas en dirección a los magnos propósitos declarados. Particularmente polémicas han sido las diferentes versiones presentadas para enfrentar la cuestión del financiamiento. En breve síntesis, ha sido propuesto crear un Fondo Solidario de Salud cuyo diseño todavía no está claro pero que, en principio, estaría formado por aportes fiscales, por las contribuciones del 7\%, y más un porcentaje de esta contribución a ser extraído del $7 \%$ de las personas que se afilian a una Isapre y elevación general del techo legal de la renta para el descuento. Sería, en realidad, un seguro que, en contrapartida, ofrecería una cesta básica universal (plan de prestaciones), al tiempo que se abre la posibilidad de que el FONASA compre servicios del sector privado para ampliar la oferta.

Si, por una parte, la propuesta de Reforma es bastante compleja, por otra, la población no parece comprometida o preocupada. Pero en- tre los grupos de interés del sector sí viene siendo muy discutida, en particular por los defensores del sector estatal, que la consideran abierta tentativa de institucionalizar de una vez la privatización total del sistema de salud, haciéndolo aun más injusto. De acuerdo con el documento Salud para todos, con todos, La Reforma Solidaria del sector salud queChile necesita, divulgado en noviembre de 2001 por la recién creada Asamblea Ciudadana por una Reforma Solidaria, todo el contenido de la Reforma debe ser objetado, centrando las críticas en la peligrosa idea de un "seguro", porque es inadmisible en el sector público, no ofrece cualquier garantía, y contribuye a la inequidad. Frentes opositores como estos y las el ecciones parlamentarias de diciembre de 2001 fueron poderosos motivos para que las autoridades ministeriales decidieran postergar la discusión de la Reforma hasta mediados del 2002.

\section{Consideraciones finales}

A lo largo de la exposición acompañamos la evolución de las políticas de salud y los formatos institucionales resultantes, teniendo como telón de fondo las coyunturas políticas en las que fueron forjadas. En esta trayectoria, aparece como un rasgo crucial la división político ideológica que ha atravesado la historia moderna del país. División en tres tercios, cuya implacable matemática impone alianzas, bien de derecha, bien de izquierda, con o sin el centro, para avanzar en proyectos destinados a cambiar el país, fundamentados primero en el Estado, luego en el mercado y, en la "neodemocracia", en un híbrido de ambos. La arquitectura del sistema de salud reflejó, a lo largo del tiempo, tanto esos movimientos como el arraigado sesgo de clase. Los productos de los embates por las reformas fueron: el estatizado SNS, en 1952, destinado a los operarios e indigentes; la introducción en el SNS de mecanismos propios de la medicina liberal, en 1968, para atender las categorías de empleados; la derrota de la iniciativa del Presidente Allende de crear un sistema universal estatal; la creación del SNSS, en 1979, bajo la dictadura, y el cortejo de transformaciones neoliberales que abrieron el mercado en la salud e institucionalizó las desigualdades entre ciudadanos; y, por último, en la "neodemocracia”, las oscilantes medidas de los gobiernos para reducir las desigualdades, consiguiendo, sin embargo, progresos innegables en términos del país y del sector.

En todos los casos, fueron evidentes las limitaciones políticas para realizar las "revolu- 
ciones" anheladas por los grupos más radicales, primero en el sentido de la socialización absoluta de la medicina, después en términos de su total privatización. En la vigencia de la democracia, los partidos políticos tuvieron un papel fundamental, decidiéndose en el voto parlamentario de la mayoría hasta dónde se podría llegar en los cambios, anteponiendo el compromiso partidario a las reales demandas de la sociedad.

El sistema de partidos de espectro tripartito en lo ideológico y programático, atravesó la vida nacional y "colonizó" las organizaciones sociales, alcanzando, como no podría dejar de ser, a la corporación médica. Ambigua por naturaleza, la profesión vivió dilemas que marcaron profundamente su identidad, traducida en doble lealtad: con los intereses corporativos y con su "vocación de servicio público". Maltratada por la dictadura, la categoría vio al grupo de ultraderecha, insignificante hasta entonces, apoderarse de la dirección de la Orden y apoyar el desmonte del emblemático SNS. Pero la privatización recolocó la contradicción inherente a la ideología de la medicina liberal, emergiendo nuevamente desde el momento en que se anunciaba la reforma en debate: por un la- do, defender transformaciones que estrechen el sector privado puede significar pérdidas en términos de mercado de trabajo y de buenos honorarios; por otro, existe el declarado compromiso ético con el bien común.

Las tensiones que vive el sector salud de Chile reflejan, en resumen, la permanencia de proyectos antagónicos alineados con la polarización ideológica del país, dividiendo políticos, dirigentes y profesionales: volver a la medicina socializada, a los tiempos del SNS, o convertir al FONASA en una gran Isapre. El proyecto de Reforma de la Salud retrata esa ambigüedad, hasta el punto de haber sido declarada una tregua en las discusiones. Y tal vez no podría ser diferente, si se considera la fragilidad de la actual coalición gobernante: en la contienda presidencial que adjudicó el mandato a Lagos para el período 2000-2006, la Concertación ganó de las fuerzas pinochetistas por apenas 2,6\% de los votos, reflejando así una clara derechización del país. Dado ese escenario político radicalizado, es poco probable que la mentada Reforma sectorial avance por cauces tranquilos en su intento de introducir realmente mayores grados de equidad en la asistencia a la salud de todos los chilenos.

\section{Referencias}

ALLENDE, S., 1939. La Realidad Médico-social Chilena. Santiago de Chile: Ministerio de Salubridad.

ASAMBLEA CIUDADANA POR UNA REFORMA SOLIDARIA, 2001. Salud para Todos, con Todos. Documento para discusión. Santiago de Chile: Asamblea Ciudadana por una Reforma Solidaria. (mimeo.)

CHILE, 1951. Historia Fidedigna de la Ley № 10.223. Crea el Estatuto delos Médicos Funcionarios. Santiago de Chile: Cámara de Diputados.

CHILE, 1952. Historia Fidedigna de la Ley № 10.383. Modifica el Seguro Social Obligatorio y crea el Servicio Nacional de Salud. Santiago de Chile: Cámara de Diputados.

CHILE, 1968. Historia Fidedigna dela Ley № 16.781. Ley de Medicina Curativa. Santiago de Chile: Cámara de Diputados. 
ELTER, D., 1999. Sistema de A.F.P. Chileno. Injusticia de un Modelo. Santiago de Chile: LOM Ediciones/ Universidad Arcis.

FAZIO, H., 1996. El Programa Abandonado. Balance Económico del Gobierno de Aylwin. Santiago de Chile: ARCIS-CENDA.

GONZALEZ-DAGNINO, A., 1999. La meta sanitaria para Chile en el 2010. Cuadernos Médico-Sociales, 40:36-50.

LABRA, M. E., 1985. O Movimento Sanitarista nos Anos 20: Da "Conexão Sanitária Internacional" à Especialização em Saúde Pública no Brasil. Dissertação de Mestrado, Rio de Janeiro: Escola Brasileira de Administração Pública, Fundação Getúlio Vargas.

LABRA, M. E., 1995. As políticas de saúde no Chile: Entre a razão e a força. In: Sistemas de Saúde. Continuidades e Mudanças (P. Buss \& M. E. Labra, org.), pp. 103-152, Rio de Janeiro: Editora Fiocruz/São Paulo: Editora Hucitec.

LABRA, M. E., 1997. Política, Saúde e Interesses Médicos no Chile (1900-1990). Tese de Doutorado, Rio de Janeiro: Instituto Universitário de Pesquisas do Rio de Janeiro.

LABRA, M. E., 2000. Padrões de formulação de políticas de saúde no Chile no século XX. Dados, 43: 153-182.

LAVADOS, I., 1984. Evolución de las Políticas Sociales en Chile 1964-1980. Santiago de Chile: Comisión Económica para América Latina.

MEDINA, E., 1989. Reajustes y orientaciones del sector salud. In: IX Jornadas Chilenas de Salud Pública, Anales, pp. 32-33. Santiago de Chile: Escuela de Salud Pública, Universidad de Chile.

MINISTERIO DE SALUD PUBLICA, 1970. Recursos Humanos de Salud en Chile. Un Modelo de Análisis. Santiago de Chile: Ministério de Salud Pública.

MINSAL (Ministerio de Salud), 1975. Sistema Nacional deServicios deSalud. Santiago de Chile: MINSAL. (mimeo.)

MINSAL (Ministerio de Salud), 1991. Situación y Atención dela Salud en Chile. Santiago de Chile: MINSAL.

MINSAL (Ministerio de Salud), 1992. Situación de Salud. Santiago de Chile: MINSAL.

MINSAL (Ministerio de Salud), 1993. Situación y Atención dela Salud en Chile. Santiago de Chile: MINSAL.
MINSAL (Ministerio de Salud), 1999. Sistematización deEstudios dela Red Asistencial deSalud. Informe País. Santiago de Chile: MINSAL. (mimeo.)

MINSAL (Ministerio de Salud), 2000. Políticas de Salud y Metas Ministeriales. Santiago de Chile: MINSAL.

MINSAL (Ministerio de Salud), 2001a. Situación de Salud. Indicadores Básicos. Santiago de Chile: MINSAL.

MINSAL (Ministerio de Salud), 2001b. Reforma del Sistema de Salud. Santiago de Chile: MINSAL.

MINSAL (Ministerio de Salud), 2001c. Inversiones Sectoriales. Estadísticas. Santiago de Chile: MINSAL.

MONTOYA, C.; MADRID, S. \& BARILARI, E., 1996. Experiencia de un camino para vencer la inequidad en salud: El enfoque del sistema público hacia las comunas menos favorecidas. Cuadernos MédicoSociales, 37:66-69.

NOVOA, S., 2001. Salud por Fonasa? El Mercurio, Santiago de Chile, 17 nov. Cuaderno D - Enfoques, pp. 1-3.

PIÑERA, J., 1991. El Cascabel al Gato. La Batalla por Ia Reforma Previsional. Santiago de Chile: Editora Zig-Zag.

RACZYNSKI, D., 1991. 'Estado de Bienestar' y Políticas Sociales en Chile: Origen, Transformaciones y Perspectivas. Documento para comentarios. Santiago de Chile: Centro de Estudios de Planificación Nacional.

RIQUELME, S. \& BARILARI, E., 1997. Equidad y salud desde una perspectiva de género. Cuadernos Mé dico-Sociales, 38:55-65.

ROZAS, M. P., 1992. Evolución del gasto social en Chile. In: El Modelo Económico Chileno (D. Wisercarver, org.), pp. 171-225, Santiago: Centro Internacional para el Desarrollo Económico.

SCHMITTER, P. C., 1993. La consolidación de la democracia y la representación de los grupos sociales. Revista Mexicana de Sociología, 3:3-31.

ZAPATTA, F., 1999. Mitos y Realidades do Sistema Privado de Fondos de Pensiones en Chile (AFP). Santiago de Chile: LOM Ediciones.

Recebido el 8 de junio de 2001

Versión final presentada el 30 de noviembre de 2001

Aprobado el 11 de marzo de 2002 